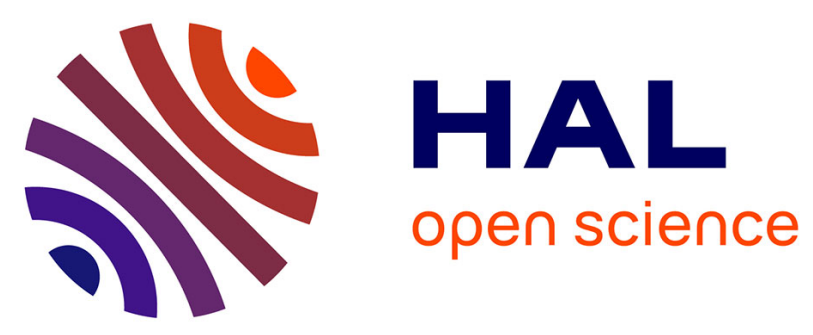

\title{
Hydration and Ion Pairing in Aqueous Mg 2+ and Zn 2+ Solutions: Force-Field Description Aided by Neutron Scattering Experiments and Ab Initio Molecular Dynamics Simulations
}

Elise Duboué-Dijon, Philip E Mason, Henry Fischer, Pavel Jungwirth

\section{To cite this version:}

Elise Duboué-Dijon, Philip E Mason, Henry Fischer, Pavel Jungwirth. Hydration and Ion Pairing in Aqueous Mg 2+ and Zn 2+ Solutions: Force-Field Description Aided by Neutron Scattering Experiments and Ab Initio Molecular Dynamics Simulations. Journal of Physical Chemistry B, 2017, 122 (13), pp.3296-3306. 10.1021/acs.jpcb.7b09612 . hal-02104585

\section{HAL Id: hal-02104585 \\ https://hal.science/hal-02104585}

Submitted on 19 Apr 2019

HAL is a multi-disciplinary open access archive for the deposit and dissemination of scientific research documents, whether they are published or not. The documents may come from teaching and research institutions in France or abroad, or from public or private research centers.
L'archive ouverte pluridisciplinaire HAL, est destinée au dépôt et à la diffusion de documents scientifiques de niveau recherche, publiés ou non, émanant des établissements d'enseignement et de recherche français ou étrangers, des laboratoires publics ou privés. 


\title{
Hydration and Ion Pairing in Aqueous $\mathrm{Mg}^{2+}$ and $\mathrm{Zn}^{2+}$ Solutions: Force Field Description
}

\section{Aided by Neutron Scattering Experiments and}

\section{Ab Initio Molecular Dynamics Simulations}

\author{
Elise Duboué-Dijon, ${ }^{*, \dagger}$ Philip E. Mason, ${ }^{*}{ }^{\dagger}$ Henry E. Fischer, ${ }^{\ddagger}$ and Pavel \\ Jungwirth*,† \\ $\dagger$ Institute of Organic Chemistry and Biochemistry, Czech Academy of Sciences, Flemingovo \\ nam. 2, 16610 Prague 6, Czech Republic \\ $\ddagger$ Institut Laue-Langevin, 71 Avenue des Martyrs, CS 20156, 38042 Grenoble Cedex 9, \\ France
}

E-mail: elise.duboue-dijon@uochb.cas.cz; philip.mason@uochb.cas.cz;

pavel.jungwirth@uochb.cas.cz 


\begin{abstract}
Magnesium and zinc dications possess the same charge and have an almost identical size. Yet, they behave very differently in aqueous solutions and play distinct biological roles. It is thus crucial to identify the origins of such different behaviors and to assess to what extent they can be captured by force field molecular dynamics simulations. In this work, we combine neutron scattering experiments in a specific mixture of $\mathrm{H}_{2} \mathrm{O}$ and $\mathrm{D}_{2} \mathrm{O}$ (the so-called null water) with ab initio molecular dynamics simulations in order to probe the difference in the hydration structure and ion pairing properties of chloride solutions of the two cations. The obtained data are used as a benchmark to develop a scaled charge force field for $\mathrm{Mg}^{2+}$ that includes electronic polarization in a mean field way. We show that using this electronic continuum correction we can describe aqueous magnesium chloride solutions well. However, in aqueous zinc chloride specific interaction terms between the ions need to be introduced to capture ion pairing quantitatively.
\end{abstract}

\title{
Introduction
}

Magnesium(II) and zinc(II) are both closed shell divalent cations with almost the same ionic radii (0.72 vs $0.74 \AA),{ }^{1}$ with the hydration free energy of $\mathrm{Zn}^{2+}$ being slightly more negative than that of $\mathrm{Mg}^{2+} .{ }^{2,3}$ Structural experimental and computational studies suggest an octahedral hydration for $\mathrm{Mg}^{2+}$ with six water molecules in the solvent shell, while the hydration number is found experimentally to be slightly smaller (5.3) in non-ion-pairing zinc triflate solutions. ${ }^{4}$ Computational studies have reported a roughly octahedral hydration for $\mathrm{Zn}^{2+}$ in solution, ${ }^{5,6}$ but its hydration shell has been suggested to be more flexible than that of $\mathrm{Mg}^{2+} .^{7}$

In molecular simulations using empirical force fields, where an ion is described by its charge and radius (typically via the Lennard-Jones potential) the two ions thus behave almost identically. Yet, in reality they never act interchangeably, even if they may in some biological 
situations bind to common structural motifs, most notably porphyrines ${ }^{8}$ — chlorophyll for magnesium and hemes for zinc. Typically, each of the two cations performs several unique and critical biological roles. For instance, $\mathrm{Mg}^{2+}$ plays a crucial role in the synthesis and biological function of ATP, ${ }^{9-11}$ while $\mathrm{Zn}^{2+}$ has a structural role in zinc fingers and is involved in insulin storage in pancreatic vesicles. ${ }^{12-14}$ It is, therefore, critical to establish the origins of such different behaviors and to determine whether these can be successfully captured by force field molecular dynamics (MD) simulations.

Divalent cations are known to represent a challenge for empirical force field calculations because of strong polarization and charge transfer effects. ${ }^{15-17}$ Previous works have shown that standard force fields using full integer charges on the divalent ions capture poorly ion pairing in ionic solutions. ${ }^{16,18}$ Effort has been made to refine divalent ion full charge force fields to describe both single ion and ion pair properties in halide salt solutions, ${ }^{19}$ but it was found necessary to use specific interaction parameters for the $\mathrm{Mg}^{2+}$-anion interaction instead of the usual combination rule. A promising way to improvement is the use of the Electronic Continuum Correction (ECC), which consists in taking into account the fast electronic polarization in a mean field approach. ${ }^{16,20}$ This is numerically implemented by scaling the charges of the ions. ${ }^{20}$ This approach has been shown in previous works to considerably improve the description of ion pairing in calcium chloride ${ }^{16}$ and calcium acetate ${ }^{18}$ ionic solutions; therefore, we seek here to develop a similar ECC description for magnesium and zinc.

The strategy adopted in the present work is to characterize first the structure of concentrated $\mathrm{MgCl}_{2}$ and $\mathrm{ZnCl}_{2}$ solutions with neutron scattering in order to assess the difference in the behavior of the two ions. Electrolyte solutions have been studied by x-ray and neutron diffraction for decades (see e.g. refs $^{21-24}$ ), realizing that the sheer number of correlations in the obtained total scattering patterns (10 for a simple salt solution) makes the data difficult to interpret. This led to the development of the technique of neutron diffraction with isotopic substitution (NDIS), ${ }^{22}$ in which the total neutron scattering patterns are obtained for 
two chemically identical solutions differing only in isotopic compositions. The subtraction of these two scattering patterns yields data originating only from the hydration of the substituted nuclei. This approach has become one of the most powerful methods to gain structural insights into the behavior of ions in aqueous solution. ${ }^{4,23,25,26}$ While zinc has suitable (albeit expensive) isotopes for the NDIS technique, magnesium does not, which precludes the direct use of NDIS to compare the hydration of the magnesium and zinc ions. For this reason, we employ here a technique, ${ }^{27}$ developed by one of us, which can provide insight into the hydration of small ions using solutions prepared with null water (i.e., a mixture of $\mathrm{D}_{2} \mathrm{O}$ and $\mathrm{H}_{2} \mathrm{O}$ in proportions that cancel the scattering from hydrogens), without the need for multiple isotopes. This approach thus allows us here to directly compare structural properties of aqueous $\mathrm{MgCl}_{2}$ and $\mathrm{ZnCl}_{2}$.

The purpose of this work is to assess the quality of commonly used force fields against the newly obtained neutron scattering data and to use the latter as a benchmark for the development of ECC force fields for magnesium and zinc cations, following an analogous strategy as previously adopted for calcium. ${ }^{16,18}$ In addition, we characterize here at the molecular level the difference between the $\mathrm{Mg}^{2+}$ and $\mathrm{Zn}^{2+}$ interaction with chloride using $a b$ initio MD simulations.

\section{Methods}

\section{Experimental details}

$3 \mathrm{~m}$ solutions of magnesium and zinc chloride were prepared by direct dissolution of $\mathrm{MgCl}_{2}$ (Sigma-Aldrich $99.9 \%$ anhydrous) and $\mathrm{ZnCl}_{2}$ (Sigma-Aldrich $99.999 \%$ anhydrous) in null water, i.e., a mixture of $\mathrm{H}_{2} \mathrm{O}$ and $\mathrm{D}_{2} \mathrm{O}$ in a molar ratio 1.78:1. This specific ratio of light and heavy water ensures that the average coherent neutron scattering length of hydrogen (which is negative for ${ }^{1} \mathrm{H}$ and positive for ${ }^{2} \mathrm{H}$ ) is zero, which in practice removes any contribution of the hydrogens from the neutron signal. Null water was prepared by the direct mixing 
of $\mathrm{H}_{2} \mathrm{O}(18 \mathrm{M} \Omega)$ and $\mathrm{D}_{2} \mathrm{O}$ (Aldrich, 99.9 atom \%). The samples were loaded into a null scattering titanium/ zirconium alloy cell. Total neutron scattering patterns were obtained at a wavelength of $0.5 \AA$ on the D4C diffractometer ${ }^{28}$ at the ILL in Grenoble (France). Each sample (null water, $3 \mathrm{~m} \mathrm{MgCl}_{2}$, and $3 \mathrm{~m} \mathrm{ZnCl}_{2}$ ) was measured for 6 hours. The data $^{29}$ were corrected for multiple scattering and absorption, ${ }^{30}$ and normalized versus a vanadium standard. Three total structure factors were thus obtained, $S^{W}(\mathrm{Q}), S^{\mathrm{MgCl}_{2}}(\mathrm{Q})$ and $S^{Z n C l}(\mathrm{Q})$ (for the null water, $3 \mathrm{~m} \mathrm{MgCl}_{2}$, and $3 \mathrm{~m} \mathrm{ZnCl}_{2}$ solutions, respectively). These can be expressed as a sum of pair-wise structure factors as follows (the experimental offset being subtracted so that the defined structure factors terminate at zero at long Q):

$$
\begin{gathered}
S^{W}(Q)=0.0374 S_{O O}(Q)-0.0374 \\
S^{M g C l_{2}}(Q)=0.0337 S_{O O}(Q)+0.0120 S_{O C l}(Q)+0.0034 S_{O M g}(Q)+0.0011 S_{C l C l}(Q) \\
+0.0006 S_{C l M g}(Q)+0.00008 S_{M g M g}(Q)-0.0508 \\
S^{Z n C l_{2}}(Q)=0.0337 S_{O O}(Q)+0.0120 S_{O C l}(Q)+0.0036 S_{O Z n}(Q)+0.0011 S_{C l C l}(Q) \\
+0.0006 S_{C l Z n}(Q)+0.0001 S_{Z n Z n}(Q)-0.0511 .
\end{gathered}
$$

Here, the prefactors (in barns) are obtained from the concentration and coherent scattering length of each nucleus. ${ }^{24}$ Substraction of the null water reference provides the difference structure factors $\Delta S^{M g-W}(Q)$ and $\Delta S^{Z n-W}(Q)$. The difference is taken such that the $S_{O O}$ term would perfectly cancel if the water structure was identical in the pure water and in the salt solution:

$$
\Delta S^{M g-W}(Q)=S^{M g C l_{2}}(Q)-\frac{0.0337}{0.0374} S^{W}(Q),
$$




$$
\Delta S^{Z n-W}(Q)=S^{Z n C l_{2}}(Q)-\frac{0.0337}{0.0374} S^{W}(Q)
$$

The residual Placzek effect ${ }^{31}$ was removed through Fourier filtering (see Supporting Information) to enable direct comparison with simulation results. A most straightforward comparison between the structure of $\mathrm{MgCl}_{2}$ and $\mathrm{ZnCl}_{2}$ solutions is obtained by taking the direct difference:

$$
\Delta S^{M g-Z n}(Q)=S^{M g C l_{2}}(Q)-S^{Z n C l_{2}}(Q)
$$

The Q-space signals are Fourier transformed to obtain the corresponding signals in the rspace $-\Delta G^{M g-W}(r), \Delta G^{Z n-W}(r)$, and $\Delta G^{M g-Z n}(r)$. Windows functions were applied to the Q-space data and two spurious peaks (at 8 and $11 \AA^{-1}$ ) due to noisy detectors were deleted before the Fourier transform to limit ringing artifacts. We checked that this did not affect the peak positions (see Supporting Information). Such pre-treatment of the data was not needed for $\Delta S^{M g-Z n}(Q)$, since most of the experimental noise already canceled in the difference.

\section{Computational details}

We performed force field simulations of concentrated aqueous $3 \mathrm{~m} \mathrm{MgCl}_{2}$ and $\mathrm{ZnCl}_{2}$ solutions in order to model the neutron scattering signals, $\Delta S^{M g-W}(Q)$ and $\Delta S^{Z n-W}(Q)$. These were computed with different force fields and compared to the experimental data. The simulation box contained 5670 water molecules, 612 chloride anions, and 306 cations $\left(\mathrm{Mg}^{2+}\right.$ or $\left.\mathrm{Zn}^{2+}\right)$. A reference simulation of pure water was also performed (with a unit cell containing 2165 water molecules). All the simulations were performed with the Gromacs5.1.1 software ${ }^{32}$ in the constant temperature/constant pressure (NpT) ensemble using the Parinello-Rahman barostat $^{33}$ with a 1 ps coupling constant and the velocity rescaling thermostat with a 0.5 ps

coupling time. Periodic boundary conditions were used employing a Particle Mesh Ewald ${ }^{34}$ treatment of long range electrostatic interactions with a 12 A cutoff. Hydrogen containting bonds were constrained using the LINCS algorithm. ${ }^{35}$ The simulation box was first equili- 
brated for at least 100 ns before a 20 ns production run.

Water molecules were described using the SPC/E force field, ${ }^{36}$ while a series of different force fields was used for the ions, in order to assess their performance in reproducing the neutron data. First, we used standard non-polarizable (full charges) force fields both for the chloride ${ }^{37}$ and the cations, $\mathrm{Mg}^{2+38}$ and $\mathrm{Zn}^{2+} \cdot{ }^{39}$ It has been shown previously ${ }^{16,18,40,41}$ that, especially for divalent cations, the description of the solution structure improves significantly upon employing the so called Electronic Continuum Correction (ECC). ${ }^{16,20,40}$ This approach takes into account the fast electronic polarization in a mean field way by scaling the charges of all the ions by the inverse of the square root of the electronic part of water dielectric constant $1 / \sqrt{\epsilon_{e l}}$. The radius of each ion needs to be further slightly reduced compared to the original full charges model in order to recover the proper ion-water distances. We used in this work the previously developed ECC force field for the chloride anion, ${ }^{41}$ which was fitted against neutron scattering data of $\mathrm{LiCl}$ solutions. Two ECC force fields were developed for $\mathrm{Mg}^{2+}$ here; the first was adjusted to reproduce the $\mathrm{Mg}^{2+}-\mathrm{O}$ distance previously reported in the literature (around 2.10-2.12 $\AA^{42-44}$ ) and used for force field parametrization, ${ }^{38}$ while the second was fitted to the measured neutron scattering signal, which required the use of a smaller $\mathrm{Mg}^{2+}$ radius. A new ECC force field was also developed for $\mathrm{Zn}^{2+}$. Since it did not capture the ion pairing in the $\mathrm{ZnCl}_{2}$ solution, we additionally defined a specific $\mathrm{Zn}^{2+}-\mathrm{Cl}^{-}$ Van der Waals interaction with $\sigma_{Z n C l}=2.6 \AA$, fitted to reproduce the $a b$ initio $\mathrm{Zn}^{2+}{ }_{-} \mathrm{Cl}^{-}$ free-energy profile. The parameters of all the employed ionic force fields are summarized in Table 1. The Lorentz-Berthelot mixing rule was used to derive the mixed Lennard-Jones parameters from self-parameters.

We obtained further insight into the ion pairing properties of $\mathrm{MgCl}_{2}$ and $\mathrm{ZnCl}_{2}$ solutions by computing the free energy profiles along the cation-chloride distance using BornOppenheimer ab initio molecular dynamics (AIMD) simulations. These simulations were performed using the Quickstep module of the CP2K3.0 package ${ }^{45}$ with the hybrid Gaussian and plane waves (GPWs) method. Due to the high computational cost of such calculations, 
Table 1: Force field parameters for the different employed ion models.

\begin{tabular}{|c|c|c|c|c|}
\hline Ion & Force field & $\mathrm{q}(e)$ & $\sigma(\AA)$ & $\epsilon(\mathrm{kJ} / \mathrm{mol})$ \\
\hline \multirow{2}{*}{$\mathrm{Cl}^{-}$} & Full $^{37}$ & -1 & 4.417 & 0.4928 \\
\cline { 2 - 5 } & ECC $^{41}$ & -0.75 & 4.100 & 0.4928 \\
\hline \multirow{3}{*}{$\mathrm{Mg}^{2+}$} & Full $^{38}$ & +2 & 1.89 & 3.6610 \\
\cline { 2 - 5 } & ECC big & +1.5 & 1.55 & 3.6610 \\
\cline { 2 - 5 } & ECC small & +1.5 & 1.36 & 3.6610 \\
\hline \multirow{2}{*}{$\mathrm{Zn}^{2+}$} & Full & +2 & 1.95 & 1.046 \\
\cline { 2 - 5 } & ECC & +1.5 & 1.75 & 1.046 \\
\hline
\end{tabular}

the simulation box had to be rather small, containing one cation $\left(\mathrm{Mg}^{2+}\right.$ or $\left.\mathrm{Zn}^{2+}\right)$ and one chloride together with 64 water molecules. The box sizes were determined from NpT simulations using the ECC classical force fields. All the AIMD simulations were performed in the NVT ensemble using a velocity rescaling thermostat (CSVR) ${ }^{46}$ with a 50 fs time constant. We used the BLYP density functional ${ }^{47,48}$ with the D3M dispersion correction ${ }^{49,50}$ with a Becke-Johnson damping scheme, ${ }^{51} \mathrm{D} 3 \mathrm{M}(\mathrm{BJ})$. The same calculations were repeated with the older D2 dispersion correction, ${ }^{52}$ with the results being qualitatively consistent. (see Supporting Information). We used the DZVP-MOLOPT-SR-GTH double- $\zeta$ basis set, ${ }^{53}$ optimized for the condensed phase, with GTH pseudopotentials ${ }^{54}$ for the core electrons and a 400 Ry cutoff for the auxiliary plane wave basis.

The free energy profile along the cation-chloride distance was obtained using $23\left(\right.$ for $\mathrm{Mg}^{2+}$ ) and 27 (for $\mathrm{Zn}^{2+}$ ) umbrella sampling windows, the cation-chloride distance being restrained in each window by a harmonic potential around a fixed value moving from $2.3 \AA$ to $6.0 \AA$. The initial configuration in each window was taken from the same umbrella sampling window equilibrated using a scaled charge force field. Each window was equilibrated for 5 ps before a 40 ps production run. A single free energy profile thus accumulates about 1 ns of sampling. Within such a timescale, given the very slow water exchange in the ion hydration shell, ${ }^{55}$ the system does not have time to explore all the possible hydration geometries. In the barrier region between the contact and solvent-shared ion pair, where the number of water molecules in the cation hydration shell is expected to go from 5 to 6 , we thus used several starting 
points with different typical hydration geometries. The free energy profile was reconstructed from the biased umbrella sampling windows using the WHAM algorithm. ${ }^{56}$ Error bars on the obtained profile were estimated by dividing each window in 8 blocks, independently evaluating the free energy profile on each block, and finally using as the estimate error the standard deviation (aligning the profiles at the top of the barrier) for the 8 blocks.

\section{Results and Discussion}

\section{Neutron scattering from concentrated $\mathrm{MgCl}_{2}$ and $\mathrm{ZnCl}_{2}$ solutions}

We obtained neutron scattering signals for $3 \mathrm{~m} \mathrm{MgCl}_{2}$ and $\mathrm{ZnCl}_{2}$ solutions in null water as well as for a pure null water sample (Fig. 1). The total structure factors $S^{M g C l}(Q)$ and $S^{Z n C l_{2}}(Q)$ are dominated by water oxygen-oxygen correlations, with only a minor fraction of the signal corresponding to correlations involving the divalent cation (Eqs. 2 and 3). Subtraction of the null water reference (Eqs. 4-5) allows us to remove most of the water O-O correlation from the experimental signal. The only remaining water O-O contributions originate from the difference in water structure between pure water and the salt solutions, and thus characterize the influence of the salt on the surrounding water. The obtained difference structure factors, $\Delta S^{M g-W}(Q)$ and $\Delta S^{Z n-W}(Q)$ (Fig. 2a), offer a very straightforward characterization of the hydration structure of $\mathrm{Mg}^{2+}$ and $\mathrm{Zn}^{2+}$. Differences between the hydration structures of $\mathrm{Mg}^{2+}$ and $\mathrm{Zn}^{2+}$ are readily apparent in Q-space in the shape and frequency of the low-Q signal. These differences translate in r-space into markedly different peak positions and shape. The r-space signal for the $\mathrm{MgCl}_{2}$ solution is mainly composed of two sharp peaks, located at $2.05 \AA$ and $3.11 \AA$, and of a smaller well-defined peak at $4.15 \AA$. In contrast, the first peak for the $\mathrm{ZnCl}_{2}$ solution is broader and shifted to $2.15 \AA$, while the second peak is very broad, extending between 2.9 and $3.9 \AA$. These features are likely due to significant ion pairing in the zinc chloride solution, as previously suggested. ${ }^{4,57,58}$

In contrast, experimental studies ${ }^{38,44}$ show no sign of contact ion pairs between magne- 


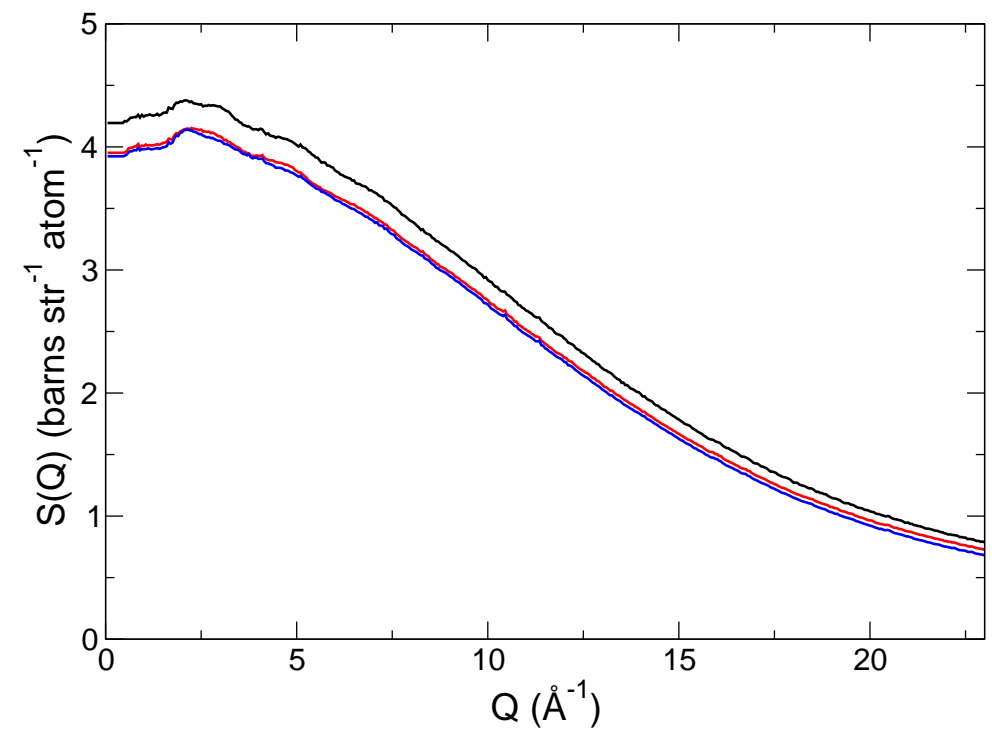

Figure 1: Raw total structure factor for null water $S^{W}(Q)$ (black), $3 \mathrm{~m} \mathrm{MgCl}_{2}$ in null water $S^{M C_{2}}(Q)$ (red) and $3 \mathrm{~m} \mathrm{ZnCl}$ in null water $S^{Z n C l_{2}}(Q)$ (blue).

sium and chloride ions in $\mathrm{MgCl}_{2}$ solutions; the first peak of the neutron signal $\Delta G^{M g-W}(r)$ can thus be readily assigned to the $\mathrm{Mg}-\mathrm{O}$ distance. The presently obtained value of $2.05 \AA$ falls within the range of experimental values, 2.0-2.12 $\AA$, previously reported in the litterature, ${ }^{42-44,59,60}$ but is shorter than the most recent neutron scattering estimate ${ }^{44}$ of $2.10 \AA$. The position of the first peak is found here to be rather insensitive to the employed signal treatment. Namely, the use of different smoothing and window functions before the Fourier transform leads in r-space to peak positions in a narrow range of $2.03-2.05 \AA$. We note that while $\operatorname{Ref}^{44}$ fits the total neutron scattering pattern, which is dominated by water-water contributions and where only a small fraction of the signal pertains to $\mathrm{Mg}-\mathrm{O}$ correlations, our present experiment offers a more direct estimate of the $\mathrm{Mg}^{2+}-\mathrm{O}$ distance.

Direct comparison between the $\mathrm{MgCl}_{2}$ and $\mathrm{ZnCl}_{2}$ solution structures is made possible by taking the difference between the two raw signals. This procedure allows us to cancel most of the experimental noise so that the obtained $\Delta S^{M g-Z n}(Q)$ (Eq. 6 and Fig 2c) is a very direct characterization of the difference between the $\mathrm{Mg}^{2+}$ and $\mathrm{Zn}^{2+}$ hydration. The data is 

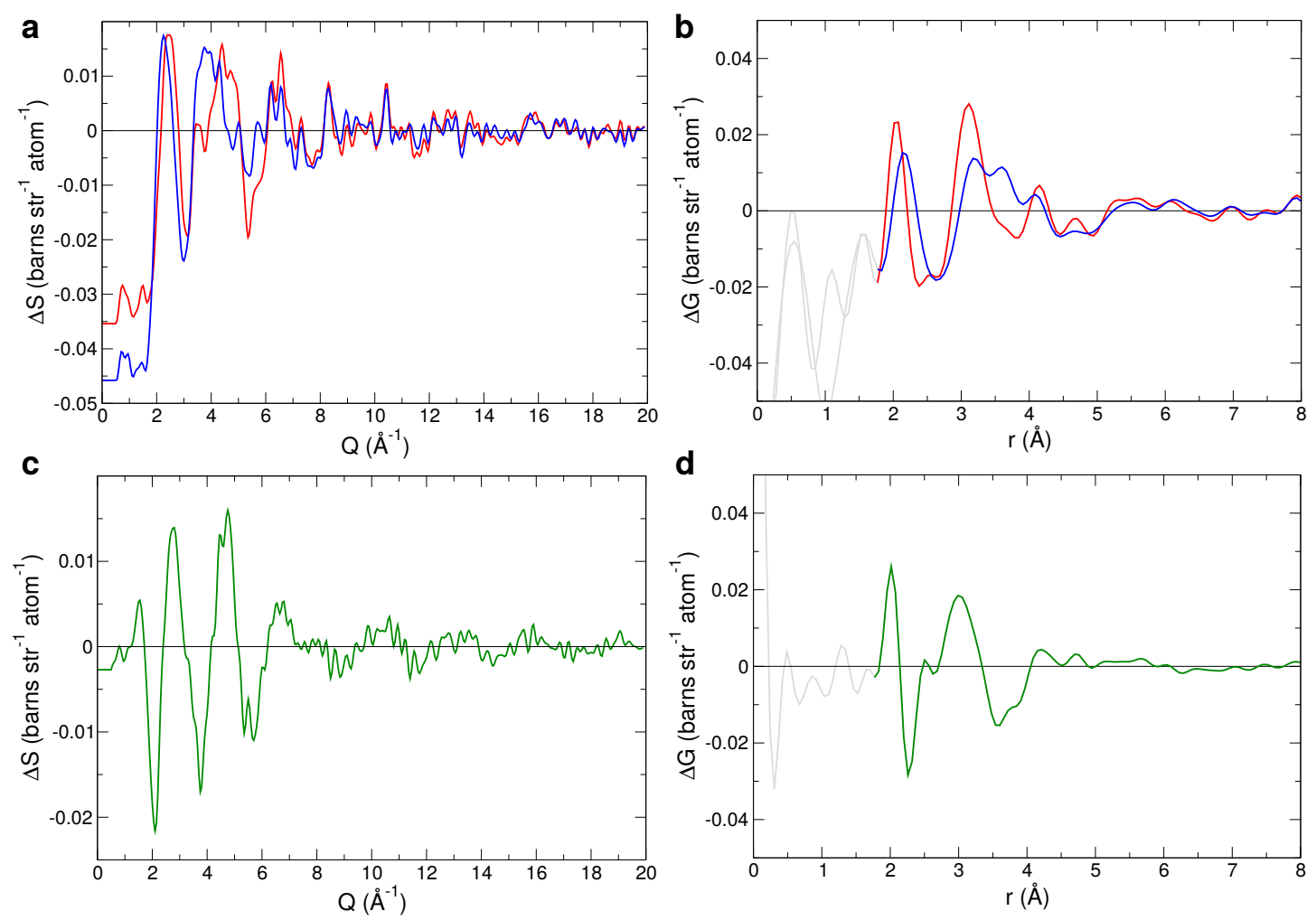

Figure 2: a) Difference structure factor $\Delta S^{M g-W}(Q)$ (red) and $\Delta S^{Z n-W}(Q)$ (blue), together with b) the corresponding Fourier-transformed r-space signals $\Delta G^{M g-W}(r)$ (red) and $\Delta G^{Z n-W}(r)$ (blue). c) Direct difference structure factor $\Delta S^{M g-Z n}(Q)$ and d) the corresponding r-space signal $\Delta G^{M g-Z n}(r)$.

so smooth that no signal treatment is needed to perform the Fourier-transform and obtain the r-space signal (Fig 2d). The resulting signal clearly shows a sharp positive peak around $2.05 \AA$ and a sharp negative peak around $2.25 \AA$.

\section{Refining $\mathrm{Mg}^{2+}$ and $\mathrm{Zn}^{2+}$ ECC force fields}

Comparison of the experimental neutron scattering patterns to those computed from molecular simulations forms a stringent test of the quality of the force fields used in molecular simulations. In particular, since most of the water-water correlations are canceled out in the first order difference, it represents a very sensitive probe of the ions' hydration. We now use this data as a benchmark for the development of scaled-charges ECC descriptions of 
$\mathrm{Mg}^{2+}$ and $\mathrm{Zn}^{2+}$. Note that such approach has been previously shown ${ }^{18}$ to better describe the interaction of aqueous calcium with biologically relevant anions.

In figure 3 we compare the experimental neutron scattering signal $\Delta S^{M g-W}(Q)$ with that computed from MD simulations of the same concentrated system using different force fields (the experimental resolution is applied to the computed signal for a fair comparison). We first use a standard force field (Fig. 3a), where ions bear the usual integer charges (i.e., +2 for $\mathrm{Mg}^{2+}$ and -1 for $\mathrm{Cl}^{-}$). The shape of the simulated signal is qualitatively similar to the experimental one. It exhibits two sharp peaks - the first one corresponding to the $\mathrm{Mg}-\mathrm{O}$ correlation, the second one to the remaining $\mathrm{O}-\mathrm{O}$ correlations and the $\mathrm{O}-\mathrm{Cl}$ correlations - and a third smaller peak at larger distance, which can be very clearly assigned to the correlation between two water oxygen atom in trans position around a $\mathrm{Mg}^{2+}$ cation. However, the computed Q-space signal exhibits a too high frequency compared to the experimental signal; this manifests as a phase shift. This corresponds in r-space to a significant shift to larger distances of all the peaks. We thus need to refine the original full charges force field by scaling the ionic charge and readjusting the ionic radius to obtain a scaled charge ECC force field with the proper $\mathrm{Mg}-\mathrm{O}$ distance. In a first ECC model, we tune $\sigma$ to obtain a 2.10$2.12 \AA \mathrm{Mg}-\mathrm{O}$ distance, following the estimates obtained in previous works, ${ }^{42-44}$ labeling this model as "ECC big". Fig. 3b shows that this model improves the agreement with neutron scattering compared to a full charge force field. However, the Q-space signal is still slightly out of phase and the r-space peaks are found at too large distances. Reducing the $\mathrm{Mg}^{2+}$ van der Waals radius $\sigma$ by about $12 \%$ ("ECC small") allows us to obtain an extremely good agreement with the experimental signal both in Q- and r-space (Fig. 3c).

We follow the same parametrization strategy for the $\mathrm{Zn}^{2+}$ ion, which is at first sight structurally very similar to $\mathrm{Mg}^{2+}$ (i.e., they possess the same charge and practically the same ionic radius). Fig. 4a demonstrates that the standard full charges description does not capture any of the specificities of the experimental $\mathrm{ZnCl}_{2}$ neutron scattering pattern. Rather, the computed r-space signal is composed of two sharp peaks and a third smaller 

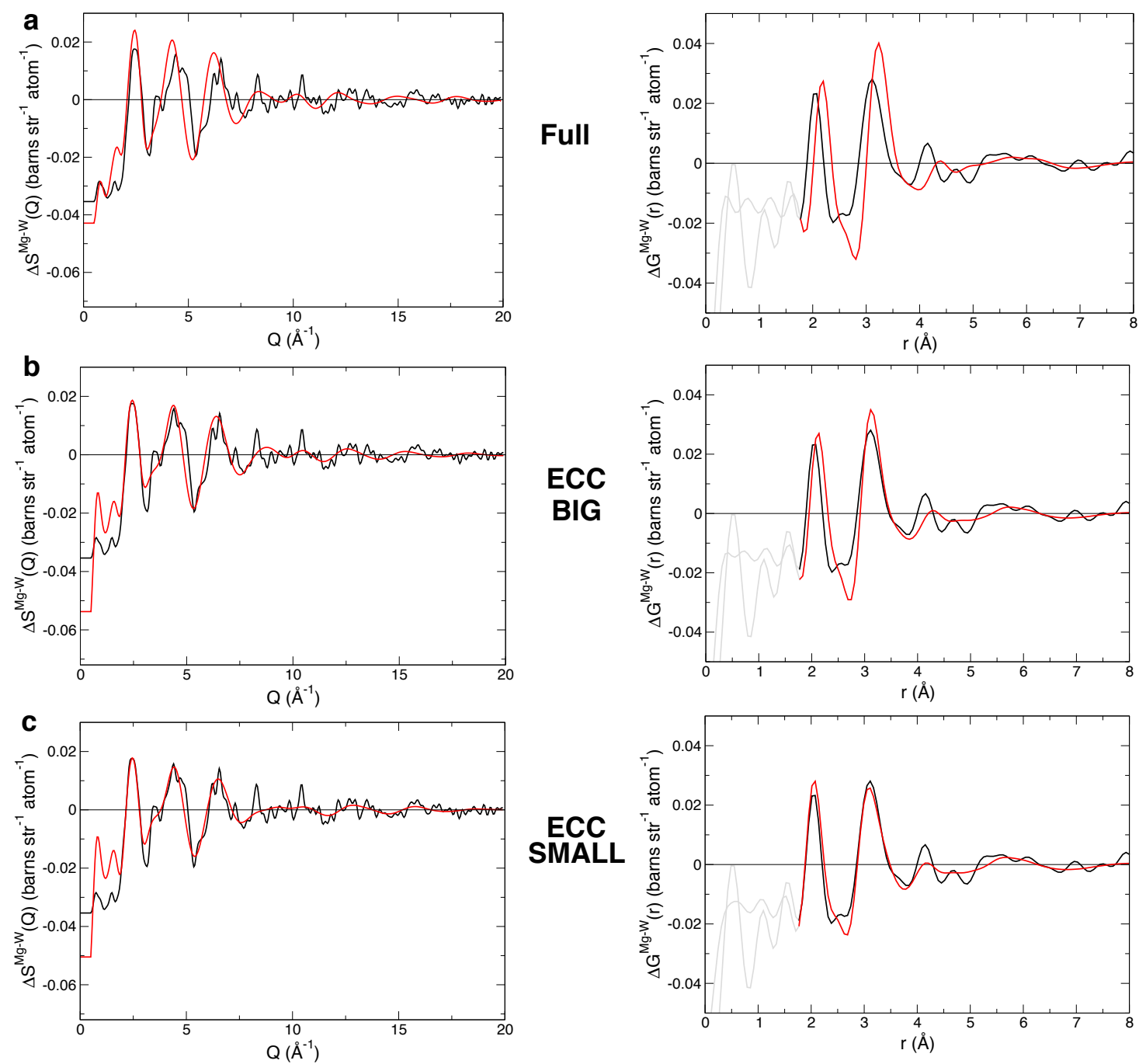

Figure 3: Comparison between the experimental (black) direct difference structure factor $\Delta S^{M g-W}(Q)$ in Q-space (left hand side) and $\Delta G^{M g-W}(r)$ in r-space (right hand side) with that obtained with a) the full charges, b) the "ECC big" and c) the "ECC small" force field for $\mathrm{Mg}^{2+}$.

well-defined peak, very similar to what we obtained for $\mathrm{MgCl}_{2}$. The model does not capture the characteristic broadening of the peaks observed for the $\mathrm{ZnCl}_{2}$ solution, which means that classical $\mathrm{Zn}^{2+}$ force fields do not provide a good description of the zinc ion. We now use the neutron scattering data as a reference to try to develop a scaled charge ECC description of the zinc cation, reducing the ionic size to recover a Zn-O distance of $2.10 \AA$, which is in the range of experimental values found in the literature. ${ }^{4,61,62}$ In this case, the use of a scaled 
charges ECC description has almost no influence on the structure of the solution as captured by the neutron scattering signal.
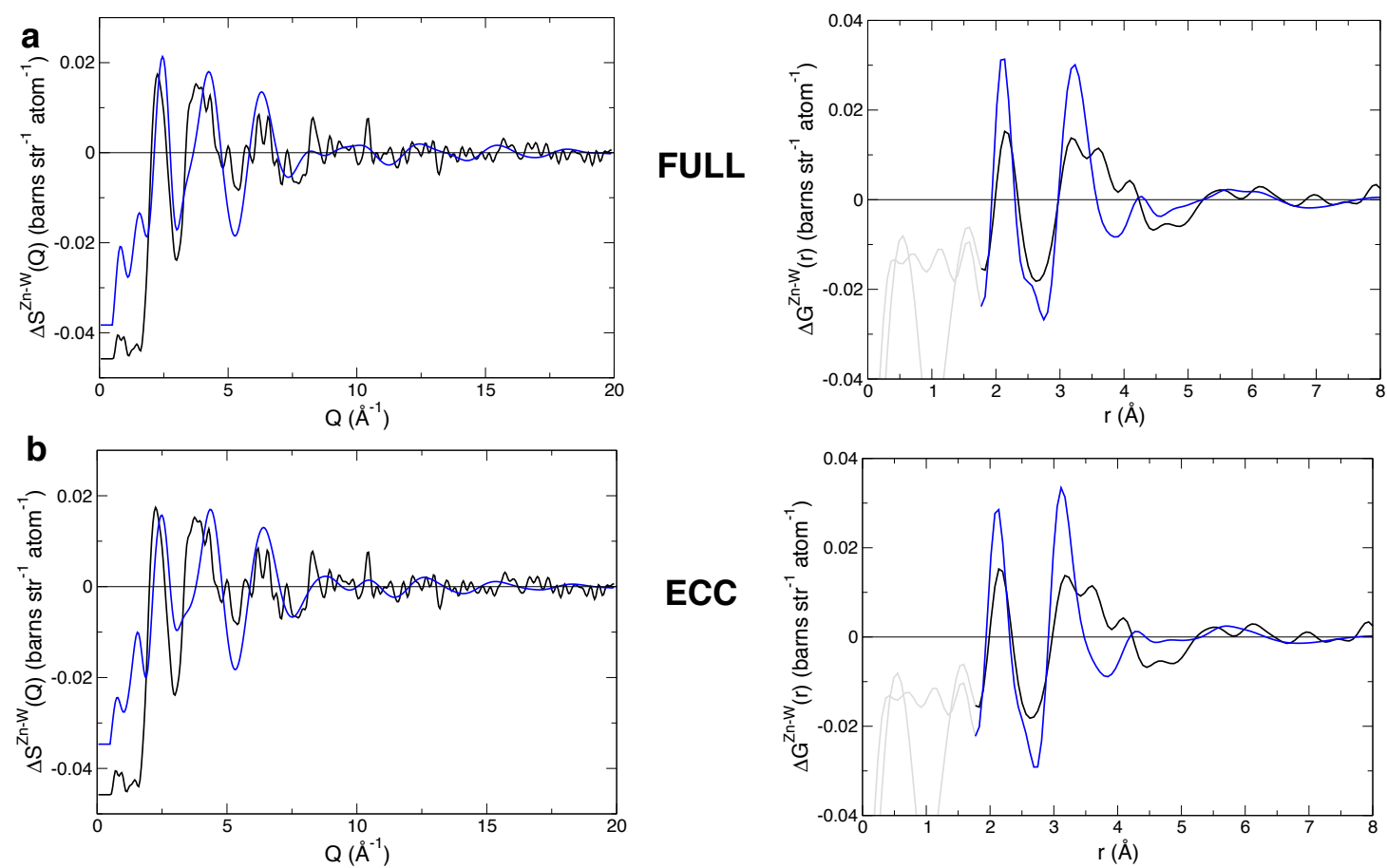

Figure 4: Comparison between the experimental (black) direct difference structure factor $\Delta S^{Z n-W}(Q)$ in Q-space (left hand side) and $\Delta G^{Z n-W}(r)$ in r-space (right hand side) with that obtained with a) the full charges and b) the "ECC" force field for $\mathrm{Zn}^{2+}$.

Several previous experimental results indicated the presence of strong ion pairing in $\mathrm{ZnCl}_{2}$ solutions. ${ }^{4,57,58}$ In contrast, none of our simulations (neither full charges nor ECC) show formation of $\mathrm{Zn}^{2+} \mathrm{Cl}^{-}$ion pairs. We further investigate this ion pairing by computing the free energy profile along the $\mathrm{Zn}^{2+}-\mathrm{Cl}^{-}$distance using ab initio MD simulations and compare it with that obtained with the above force fields (Fig. 5). We also contrast these results with those obtained for the $\mathrm{Mg}^{2+} \mathrm{Cl}^{-}$ion pair in order to identify the key differences between the aqueous behavior of the zinc and magnesium cations to be captured in the force field simulations.

Comparison of the $a b$ initio free energy profiles for the magnesium and zinc chloride ion pairs immediately reveals significant differences between $\mathrm{MgCl}_{2}$ and $\mathrm{ZnCl}_{2}$ solutions, despite 
the relatively large error bars due to short simulation times and issues with sampling different hydration geometries at the barrier region. While the $\mathrm{Mg}^{2+} \mathrm{Cl}^{-}$contact ion pair (CIP), at a interionic distance of $2.55 \AA$, is about $9 \mathrm{~kJ} / \mathrm{mol}$ higher in free energy than the solvent shared ion pair (SShIP), the $\mathrm{Zn}^{2+} \mathrm{Cl}^{-} \mathrm{CIP}$ is found at a much smaller distance of $2.25 \AA$ and is about $4 \mathrm{~kJ} / \mathrm{mol}$ more stable than the SShIP. This means that we do not expect significant contact ion pairing in $\mathrm{MgCl}_{2}$ solutions, while the formation of $\mathrm{Zn}^{2+} \mathrm{Cl}^{-}$ion pairs is strongly implied. Moreover, higher coordination zinc chloride complexes, i.e., $\mathrm{ZnCl}_{2}, \mathrm{ZnCl}_{2}^{-}, \mathrm{ZnCl}_{3}^{2-}$, and $\mathrm{ZnCl}_{4}^{3-}$ are likely to exist too, as suggested in the literature. ${ }^{4,57,58}$
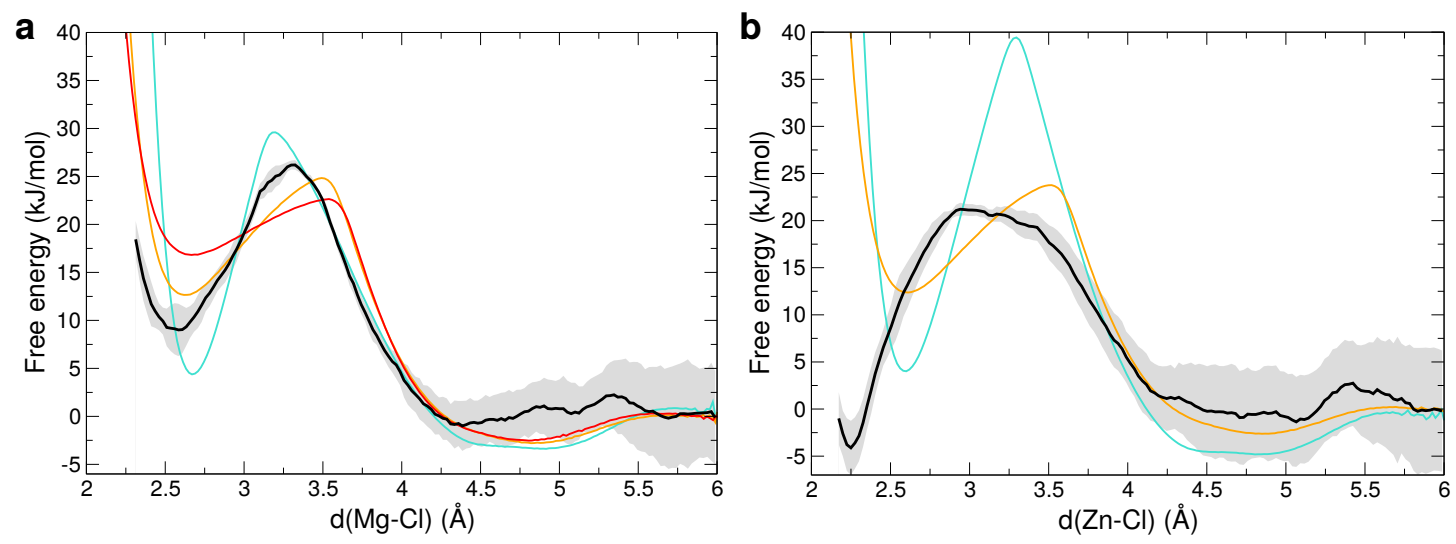

Figure 5: a) Free energy profile along the $\mathrm{Mg}^{2+}{ }_{-} \mathrm{Cl}^{-}$distance using ab initio MD (black), and the full charges (cyan), the "ECC big" (orange) and the "ECC small" (red) force fields. b) Free energy profile along the $\mathrm{Zn}^{2+}{ }_{-} \mathrm{Cl}^{-}$distance using ab initio $\mathrm{MD}$ (black), and the full charges (cyan) or the ECC (red) force field

All the studied $\mathrm{Mg}^{2+}$ force fields are in qualitative agreement with the AIMD reference, with all of them finding the CIP much higher in free energy than the SShIP, which thus leads to negligible contact ion pairing in concentrated solutions. Even after a long equilibration of our concentrated solutions, we do not observe a single $\mathrm{Mg}^{2+} \mathrm{Cl}^{-}$contact ion pair. The present "ECC big" $\mathrm{Mg}^{2+}$ force field provides the best agreement with the AIMD profile in terms of the barrier height and relative free energy of the CIP and SShIP. It, however, does not provide the best fit with the neutron scattering experiment, which implies the use of a smaller $\mathrm{Mg}^{2+}$. At this point it is interesting to note that the $a b$ initio MD simulation yields 
an optimal Mg-O distance of $2.10 \mathrm{~A}$ (corresponding to the first peak in the magnesium-water radial distribution function), which is consistent with previous works ${ }^{63-66}$ but larger than the $2.05 \AA$ value determined from the neutron experiment. We thus tested the influence of the employed level of electronic structure theory on the geometry of a $\mathrm{Mg}\left(\mathrm{H}_{2} \mathrm{O}\right)_{6}^{2+}$ cluster (see Supporting Information, Table S1). We found variations of up to $0.04 \AA$ of the $\mathrm{Mg}-\mathrm{O}$ distance with the level of theory and size of the basis set. These tests suggested that the BLYP-D3M(BJ) level of theory used in our AIMD simulations may slightly overestimate the $\mathrm{Mg}-\mathrm{O}$ distance; however, the use of more advanced methods is at present computationally too expensive to be employed in condensed phase simulations.

While all the employed magnesium force fields provide at least a qualitative agreement with the reference AIMD profile, none of the zinc force field correctly captures the remarkable stability of the contact ion pair. Hence, we find no contact ion pairs in our force field simulations of concentrated $\mathrm{ZnCl}_{2}$ solutions, while AIMD and experiments clearly indicating their presence. Another problem is that both force fields find an interionic distance for the (energetically unfavorable) contact ion pair around $2.6 \AA$, similar to what was obtained for magnesium but much higher than the reference AIMD distance of $2.25 \AA$. Additionally, the full charge force field overestimates the barrier between the SShIP and the CIP by about $20 \mathrm{~kJ} / \mathrm{mol}$, while this barrier is reproduced within a couple of $\mathrm{kJ} / \mathrm{mol}$ with the ECC description. Comparison with the free energy profile obtained using the fully polarizable force field AMOEBA ${ }^{67-69}$ (see Supporting Information) shows that the results obtained with the ECC and AMOEBA force fields are very similar to each other and that the specific character of the $\mathrm{Zn}-\mathrm{Cl}$ ion pairing is not captured even with an explicit description of the electronic polarization. This, together with the very short $\mathrm{Zn}-\mathrm{Cl}$ distance in the $\mathrm{CIP}$, suggests that the observed effects are due to specific electronic interactions between the ions.

We further investigate the interaction of $\mathrm{Zn}^{2+}$ with the chloride anion by analyzing the distribution of the distances between the chloride anion and the localized Wannier orbital center (WOC $)^{70}$ corresponding to the chloride-cation bond (Fig. 6a). The WOC is clearly 
located further away from the chloride (hence closer to the cation) in the zinc ion pair than in that with magnesium, which is indicative of a more covalent interaction in the former case. In addition, we note that the zinc hydration at the SShIP and further is found to be more flexible than that of magnesium, with an average zinc coordination number slightly below six (Fig. 6b). At short distances, the chloride anion replaces exactly one water molecule from the $\mathrm{Mg}^{2+}$ solvation shell, so that it remains perfectly octahedral (Fig. 6c). In contrast, the hydration number of zinc in the CIP falls down to three, with the solvation shell adopting a tetrahedral geometry (Fig. 6d). Such changes in the zinc hydration shell are not captured by any of the tested force field, which all exhibit a behavior more similar to $\mathrm{Mg}^{2+}$ than to $\mathrm{Zn}^{2+}$.
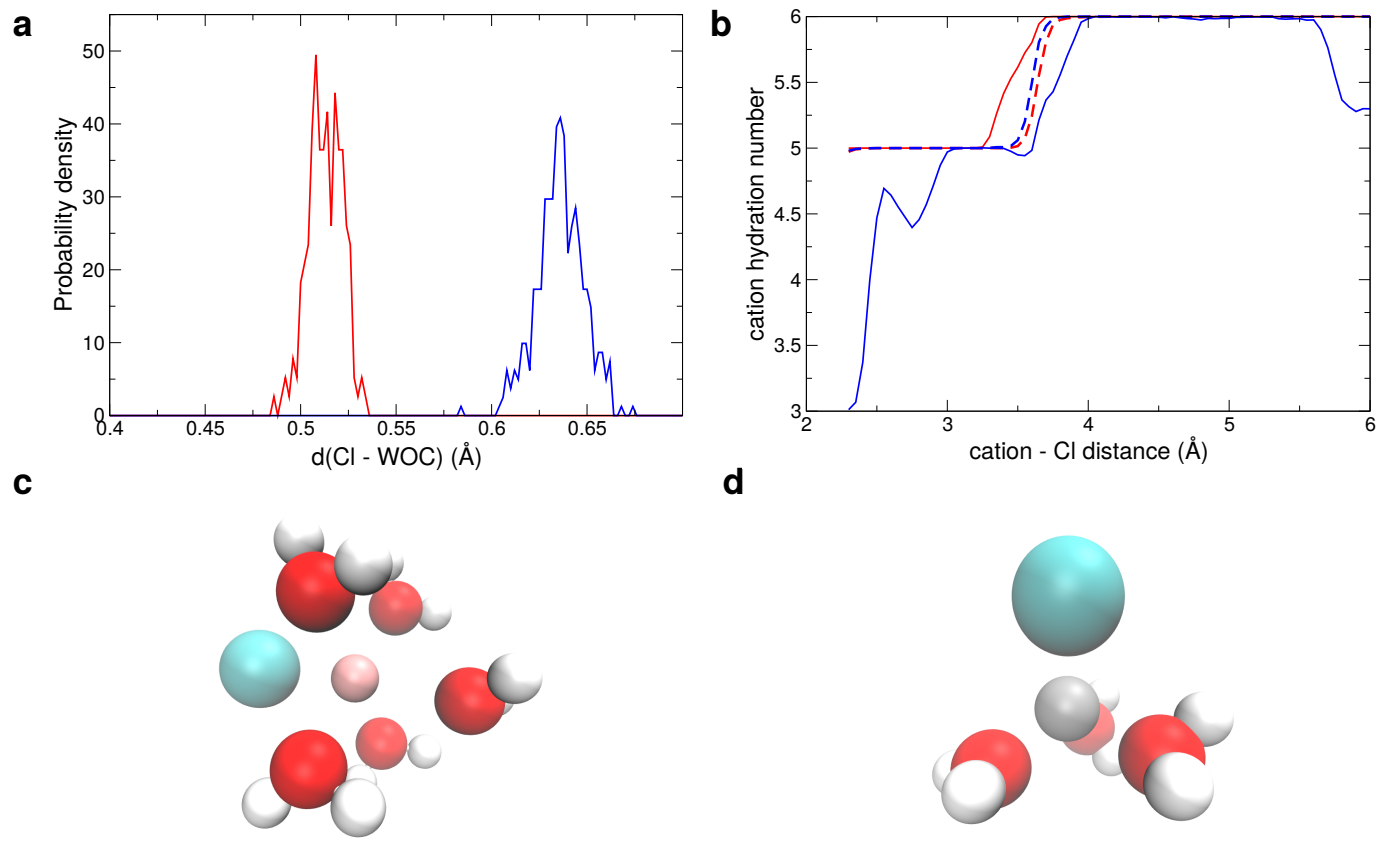

Figure 6: a) Distribution of distances between the chloride anion and the WOC describing the cation-chloride interaction. b) Hydration number (defined as the number of water molecules within $3.0 \AA$ of the cation) of $\mathrm{Mg}^{2+}$ (red) and $\mathrm{Zn}^{2+}$ (blue) as a function of the cationchloride distance, obtained from AIMD (solid lines) and ECC force field simulations (dashes). Representative snapshot of c) the magnesium chloride and d) the zinc chloride ion pairs.

In order to describe better the hydration structure and the ion pairing for zinc in the 
force field simulations, we introduce a specific Lennard-Jones interaction between zinc and chloride $\left(\sigma_{Z n C l}=2.6 \AA\right)$, which is adjusted to reproduce the AIMD free energy profile along the $\mathrm{Zn}-\mathrm{Cl}$ distance (Fig. 7a). While no ion pairs are found with the original ECC force field in a concentrated $3 \mathrm{~m} \mathrm{ZnCl}_{2}$ solution, the addition of the specific $\mathrm{Zn}-\mathrm{Cl}$ interaction leads to the formation of numerous zinc chloride complexes, with $15 \%$ of the zinc ions being involved in a $\mathrm{ZnCl}_{4}^{2-}$ complex, $9 \%$ in a $\mathrm{ZnCl}^{+}$complex, $7.5 \%$ in a $\mathrm{ZnCl}_{3}^{-}$complex, and less than $1 \%$ in a $\mathrm{ZnCl}_{2}$ complex (Fig. 7b). The computed neutron scattering signal $\Delta S^{Z n-W}(Q)$ (Fig. 8) is in much better agreement with the experiment than the original ECC force field, with a sizable broadening in the r-space of the second peak, corresponding to $\mathrm{Cl}-\mathrm{Cl}$ correlations around a zinc ion. However, the first peak remains too sharp and the second peak is still not broad enough compared to the experiment.
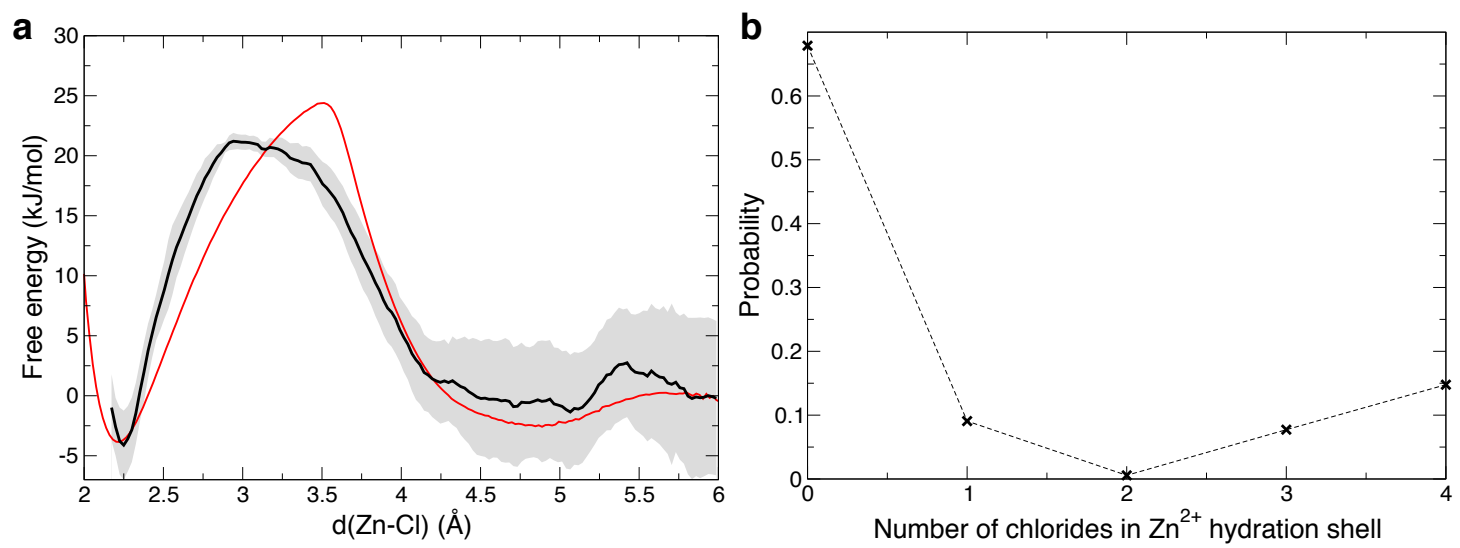

Figure 7: a) Free energy profile along the zinc chloride distance from ab initio MD simulations (black) and force field simulations using the ECC description of $\mathrm{Zn}^{2+}$ with an additional specific $\mathrm{Zn}^{2+}-\mathrm{Cl}^{-}$interaction. b) Probability distribution of different zinc chloride complexes as obtained from force field simulations with the ECC description of $\mathrm{Zn}^{2+}$ with an additional specific $\mathrm{Zn}^{2+}-\mathrm{Cl}^{-}$interaction.

As a next step, we took 10 different snapshots from a simulation using the above force field (employing a small box of 92 water molecules, $5 \mathrm{Zn}^{2+}$ and $10 \mathrm{Cl}^{-}$) where we observe a range of different zinc chloride complexes, and ran from each of these starting points a 20 ps AIMD simulation. This allowed for the relaxation of the different bond distances and of the hydration geometries of the ions. The neutron scattering signal computed from these 
10 AIMD simulations now compares extremely well with the experiment, with the proper shape and size of the first peak in r-space (Zn-O correlation) and a very broad second peak (Fig. 8b). The improvement in the neutron signal is primarly due to the relaxation of the $\mathrm{Zn}-\mathrm{Cl}$ distance, which is found to be shorter with our force field than in the AIMD simulation of the concentrated $\mathrm{ZnCl}_{2}$ solution (2.15 $\AA$ vs 2.25-2.30 $\AA$ ). Since the amount of ion pairing and nature of the observed complexes does not change within the timescale of the AIMD simulation, the extremely good agreement with the experiment shows that our $\mathrm{ECC} \mathrm{Zn}^{2+}$ force field with a specific $\mathrm{Zn}-\mathrm{Cl}$ interaction correctly captures the amount of ion pairing in the solution.
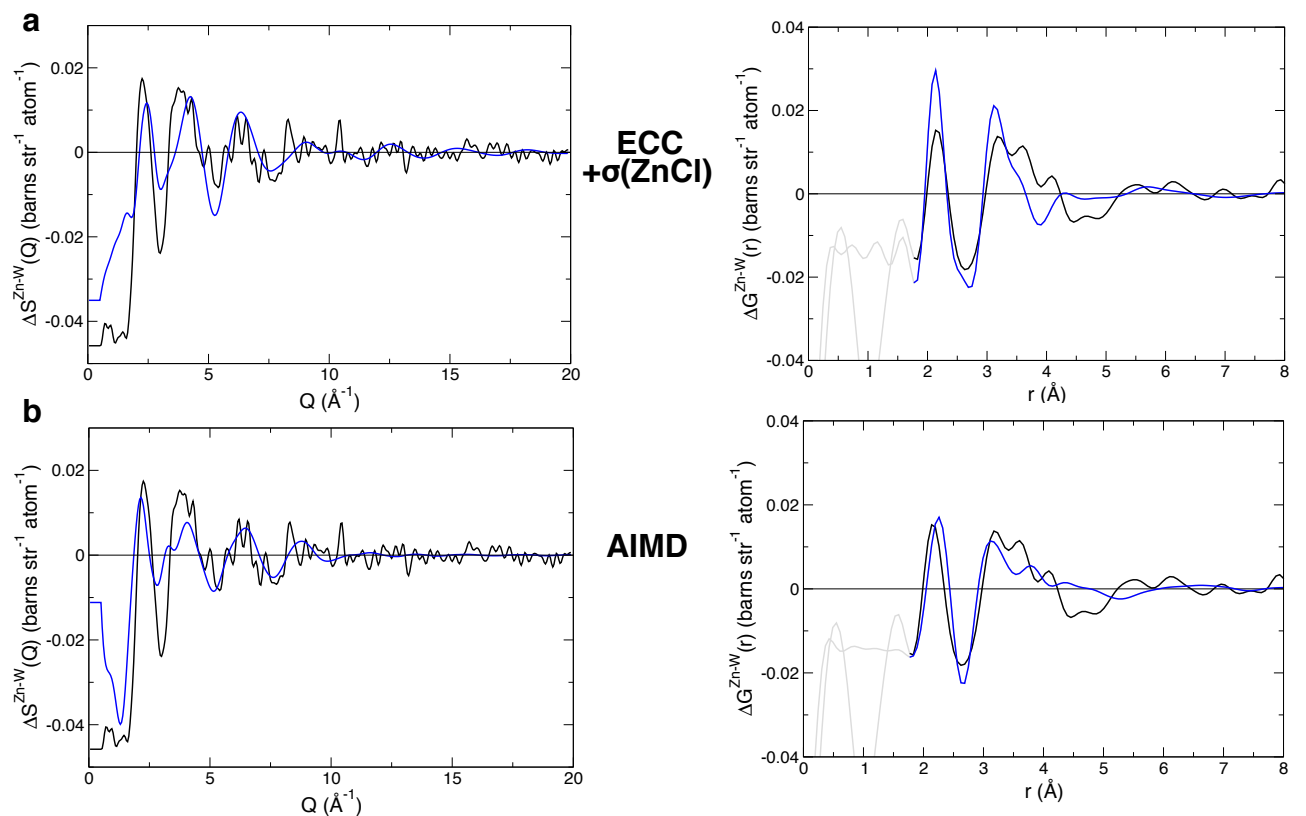

Figure 8: Comparison between the experimental (black) direct difference structure factor $\Delta S^{Z n-W}(Q)$ in Q-space (left hand side) and $\Delta G^{Z n-W}(r)$ in r-space (right hand side) with that obtained with a) the ECC force field with a specific $\sigma_{Z n C l}$ and b) after short ab initio MD equlibration of the geometries.

Finally, we compute the neutron scattering signal $\Delta S^{M g-Z n}(Q)$ using our best ECC force fields for $\mathrm{Mg}^{2+}$ and $\mathrm{Zn}^{2+}$ and compare it with the experimental data and with the signal computed with the original full charge force field (Fig. 9). As we already noted, the $\Delta S^{M g-Z n}(Q)$ and $\Delta G^{M g-Z n}(r)$ experimental signals are obtained with only minimal data 
treatment, and are thus a direct measure of the difference in solvation properties of zinc and magnesium ions. The standard force field performs extremely poorly, with a totally out of phase Q-space signal and an opposite behavior of the difference in r-space. In contrast, our best ECC force fields (i.e., "ECC small" for $\mathrm{Mg}^{2+}$ and the ECC with specific $\sigma_{Z n C l}$ for $\mathrm{Zn}^{2+}$ ) provide a good agreement with the experimental data, with proper Q-space phasing and correct peak positions in r-space.
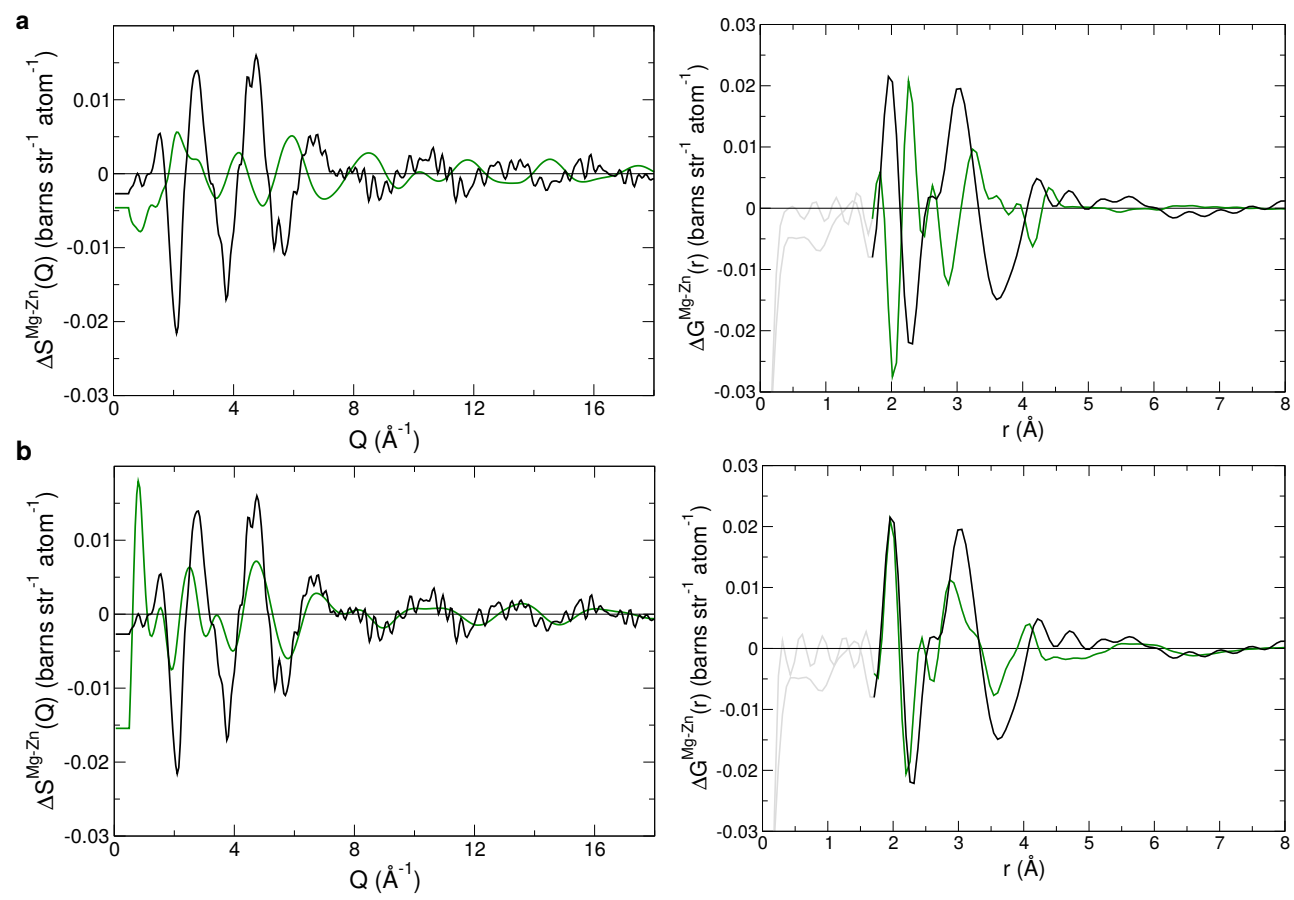

Figure 9: Comparison between the experimental (black) direct difference structure factor $\Delta S^{M g-Z n}(Q)$ in Q-space (left hand side) and $\Delta G^{M g-Z n}(r)$ in r-space (right hand side) with that obtained with a) the full charges or b) our best ECC force field ("ECC small" for $\mathrm{Mg}^{2+}$ and "ECC $+\sigma_{Z n C l}$ " for $\mathrm{Zn}^{2+}$ ).

\section{Conclusions}

In the present work we have investigated the difference in hydration and ion pairing behaviors between $\mathrm{Mg}^{2+}$ and $\mathrm{Zn}^{2+}$ cations by combining neutron scattering experiments with ab initio and force field molecular dynamics simulations. The chosen experimental strategy, with 
the use of null water solutions, allowed us to directly compare $\mathrm{MgCl}_{2}$ and $\mathrm{ZnCl}_{2}$ solution structures using exactly the same experimental setup for both cations, without the need for isotopic substitution on the cations. We took advantage of this newly obtained neutron scattering data - which notably provide a slightly smaller estimate of the Mg-O distance than previously determined - to assess the performance of standard force field in capturing the difference in behavior between $\mathrm{Mg}^{2+}$ and $\mathrm{Zn}^{2+}$ in aqueous solutions and to develop improved ECC (scaled charges) force fields for both ions. While we found that we could very well reproduce the $\mathrm{Mg}^{2+}$ experimental data with such a force field, no force field, neither standard nor ECC, could capture the specific behavior of the $\mathrm{Zn}^{2+}$ ion. We further analyzed the aqueous zinc chloride interaction with ab initio MD simulations, showing that $\mathrm{Zn}^{2+}$ forms very stable contact ion pairs with the chloride ion with a partly covalent character. The AIMD simulations also evidenced a change in the zinc hydration geometry from octahedral to tertrahedral upon ion pairing with the chloride anion. These differences are beyond polarization effects, and therefore, cannot be captured even by fully polarizable force fields. An improved description of the $\mathrm{ZnCl}_{2}$ solution is obtained upon adding a specific interaction term between zinc and chloride ions, which allows us to recover the strong ion pairing and formation of various zinc chloride complexes.

Further testing of the newly developed ECC $\mathrm{Mg}^{2+}$ force field will be performed in future studies, in particular to assess the accuracy of the $\mathrm{Mg}^{2+}$ interaction with biomolecules. Future work will also consist in systematically assessing the ion pairing properties of $\mathrm{Zn}^{2+}$ with important biological motifs (e.g., carboxylate groups or imidazole moieties). In particular, we will try to determine when the addition of a specific interaction term to the ECC force field (which was successfully tested here for the aqueous $\mathrm{ZnCl}_{2}$ solutions) is required and whether it suffices to obtain a correct description of the ion-ion and ion-water interactions. 


\section{Acknowledgement}

We are grateful to Frank Uhlig for useful discussions. We thank the staff of D4C and of the Institut Laue Langevin chemistry laboratory for their help with the neutron scattering experiments. PJ thanks the Czech Science Foundation for support via a grant no. 1601074S. EDD acknowledges support from the EMBO and Marie Curie Actions (fellowship ALTF 952-2015). This work was performed using computer time allocated by the national supercomputing center IT4innovations in Ostrava (project OPEN-8-35) and MetaCentrum data storage facilities.

\section{Supporting Information Available}

Additional information about the neutron scattering experiments, influence of dispersion correction on AIMD simulations, and comparison with the fully polarizable AMOEBA force field. This material is available free of charge via the Internet at http://pubs.acs.org/.

\section{References}

(1) Shannon, R.; Prewitt, C. Effective Ionic Radii in Oxides and Fluorides. Acta Cryst. 1969, B25, 925.

(2) Marcus, Y. Thermodynamics of Solvation of Ions. Part 5. Gibbs Free Energy of Hydration at 298.15 K. J. Chem. Soc. Faraday Trans. 1991, 87, 2995-2999.

(3) Friedman, H.; Krishnan, C. Thermodynamics of Ion Hydration in Water: A Comprehensive Treatise, Vol 3, f. franks ed.; Plenum: New York, 1973.

(4) Powell, D.; Gullidge, P.; Neilson, G.; Bellissent-Funel, M.-C. $\mathrm{Zn}^{2+}$ Hydration and Complexation in Aqueous Electrolyte Solutions. Mol. Phys. 1990, 71, 1107-1116.

(5) Fatmi, M. Q.; Hofer, T. S.; Randolf, B. R.; Rode, B. M. an Extended Ab-Initio QM/MM MD 
Approach to Structure and Dynamics of Zn(II) in Aqueous Solution. J. Chem. Phys. 2005, 123,054514 .

(6) Brancato, G.; Rega, N.; Barone, V. Microsolvation of the Zn(II) Ion in Aqueous Solution: A Hybrid QM/MM MD Approach Using Non-Periodic Boundary Conditions. Chem. Phys. Lett. 2008, 451, 53-57.

(7) Bock, C. W.; Katz, A. K.; Glusker, J. P. Hydration of Zinc Ions: A Comparison with Magnesium and Beryllium Ions. J. Am. Chem. Soc. 1995, 117, 3754-3765.

(8) Biesaga, M.; Pyrzynska, K.; Trojanowicz, M. Porphyrins in Analytical Chemistry. A Review. Talanta 2000, 51, 209-224.

(9) Ko, Y. H.; Hong, S.; Pedersen, P. L. Chemical Mechanism of ATP Synthase. J. Bio 1999, 274, $28853-28856$.

(10) Buchachenko, A. L.; Kouznetsov, D. A.; Breslavskaya, N. N.; Orlova, M. A. Magnesium Isotope Effects in Enzymatic Phosphorylation. J. Phys. Chem. B 2008, 112, 2548-2556.

(11) Pasternak, K.; Kocot, J.; Horecka, A. Biochemistry of Magnesium. J. Elementol. 2010, 15, $601-616$.

(12) Kambe, T.; Fukada, T.; Toyokuni, S. The Cutting Edge of Zinc Biology. Arch. Biochem. Biophys. 2016, 611, 1-2.

(13) Lipscomb, W. N.; Sträter, N. Recent Advances in Zinc Enzymology. Chem. Rev. 1996, 96, 2375-2434.

(14) Emdin, S. O.; Dodson, G. G.; Cutfield, J. M.; Cutfield, S. M. Role of Zinc in Insulin Biosynthesis. Diabetologia 1980, 19, 174-182.

(15) Sakharov, D. V.; Lim, C. Zn Protein Simulations Including Charge Transfer and Local Polarization Effects. J. Am. Chem. Soc. 2005, 127, 4921-4929.

(16) Kohagen, M.; Mason, P. E.; Jungwirth, P. Accurate Description of Calcium Solvation in Concentrated Aqueous Solutions. J. Phys. Chem. B 2014, 118, 7902-7909. 
(17) Soniat, M.; Hartman, L.; Rick, S. W. Charge Transfer Models of Zinc and Magnesium in Water. J. Chem. Theory Comput. 2015, 11, 1658-1667.

(18) Martinek, T.; Duboué-Dijon, E.; Timr, S.; Mason, P. E.; Boxova, K.; Pluharova, E.; Jungwirth, P. Calcium Dications in Aqueous Solutions: Accurate Force Field Description Aided by Ab Initio Molecular Dynamics Simulations and Neutron Scattering Experiments. submitted 2017 ,

(19) Mamatkulov, S.; Fyta, M.; Netz, R. R. Force Fields for Divalent Cations Based on Single-Ion and Ion-Pair Properties. J. Chem. Phys. 2013, 138, 024505.

(20) Leontyev, I.; Stuchebrukhov, A. Accounting for Electronic Polarization in Non-Polarizable Force Fields. Phys. Chem. Chem. Phys. 2011, 13, 2613-2626.

(21) Enderby, J. E. Ion Solvation Via Neutron Scattering. Chem. Soc. Rev. 1995, 24, 159-168.

(22) Finney, J. L.; Soper, A. K. Solvent Structure and Perturbation in Solutions of Chemical and Biological Importance. Chem. Soc. Rev. 1994, 23, 1-10.

(23) Neilson, G. W.; Tromp, R. H. Neutron and X-Ray Diffraction on Aqueous Solutions. Ann. Rep. Chem., Sect. C 1991, 88, 45-75.

(24) Neilson, B. G. W.; Mason, P. E.; Ramos, S.; Sullivan, D. Neutron and X-Ray Scattering Studies of Hydration in Aqueous Solutions. Phil. Trans. R. Soc. Lond. A 2001, 359, 1575-1591.

(25) Powell, H.; Barnes, A. C.; Enderby, J. E.; Neilson, G. W.; Salmon, P. S. The Hydration Structure Around Chloride Ions in Aqueous Solution. Faraday Discuss. Chem. Soc. 1988, 85, $137-146$.

(26) Badyal, Y. S.; Barnes, A. C.; Cuello, G. J.; Simonson, J. M. Understanding the Effects of Concentration on the Solvation Structure of $\mathrm{Ca}^{2+}$ in Aqueous Solution. II: Insights into Longer Range Order from Neutron Diffraction Isotope Substitution. J. Phys. Chem. A 2004, 108, $11819-11827$. 
(27) Mason, P. E.; Ansell, S.; Neilson, G. W. Neutron Diffraction Studies of Electrolytes in Null Water: A Direct Determination of the First Hydration Zone of Ions. J. Phys. Condens. Matter 2006, 18, 8437-8447.

(28) Fischer, H. E.; Cuello, G. J.; Palleau, P.; Feltin, D.; Barnes, A. C.; Badyal, Y. S.; Simonson, J. M. D4C: A Very High Precision Diffractometer for Disordered Materials. Appl. Phys. A: Mater. 2002, 74, 160-162.

(29) Mason, P.; Boxova, K.; Duboué-Dijon, E.; Fischer, H. E.; Jungwirth, P. Towards a Faithful Descripion of Biologically Relevant Dications. Institut Laue-Langevin. 2016, doi:10.5291/ILLDATA.8-05-424.

(30) Barnes, A. C.; Lague, S. B.; Salmon, P. S.; Fischer, H. E. A Determination of the Structure of Liquid $\mathrm{Ag}_{2}$ Se Using Neutron Diffraction and Isotopic Substitution. J. Phys.: Condens. Matt. 1997, 9, 6159-6173.

(31) Placzek, G. the Scattering of Neutrons by Systems of Heavy Nuclei. Phys. Rev. 1952, 86, $377-388$.

(32) Van der Spoel, D.; Lindahl, E.; Hess, B.; Groenhof, G.; Mark, A. E.; Berendsen, H. J. C. GROMACS: Fast, Flexible, and Free. J. Comput. Chem. 2005, 26.

(33) Parrinello, M.; Rahman, A. Polymorphic Transitions in Single Crystals: A New Molecular Dynamics Method. J. Appl. Phys. 1981, 52, 7182-7190.

(34) Darden, T.; York, D.; Pedersen, L. Particle Mesh Ewald: An Nlog(N) Method for Ewald Sums in Large Systems. J. Chem. Phys. 1993, 98, 10089.

(35) Hess, B.; Bekker, H.; Berendsen, H. J. C.; Fraaije, J. G. E. M. LINCS: A Linear Constraint Solver for Molecular Simulations. J. Comput. Chem. 1997, 18, 1463-1472.

(36) Berendsen, H. J. C.; Grigera, J. R.; Straatsma, T. P. The Missing Term in Effective Pair Potentials. J. Phys. Chem. 1987, 91, 6269-6271. 
(37) Chandrasekhar, J.; Spellmeyer, D. C.; Jorgensen, W. L. Energy Component Analysis for Dilute Aqueous Solutions of $\mathrm{Li}^{+}, \mathrm{Na}^{+}, \mathrm{F}^{-}$, and $\mathrm{Cl}^{-}$Ions. J. Am. Chem. Soc. 1984, 106, 903-910.

(38) Callahan, K. M.; Casillas-Ituarte, N. N.; Roeselová, M.; Allen, H. C.; Tobias, D. J. Solvation of Magnesium Dication: Molecular Dynamics Simulation and Vibrational Spectroscopic Study of Magnesium Chloride in Aqueous Solutions. J. Phys. Chem. A 2010, 114, 5141-5148.

(39) Stote, R. H.; Karplus, M. Zinc Binding in Proteins and Solution: A Simple but Accurate Nonbonded Representation. Proteins 1995, 23, 12-31.

(40) Pluharova, E.; Mason, P. E.; Jungwirth, P. Ion Pairing in Aqueous Lithium Salt Solutions with Monovalent and Divalent Counter-Anions. J. Phys. Chem. A 2013, 117, 11766-11773.

(41) Pluharova, E.; Fischer, H. E.; Mason, P. E.; Jungwirth, P. Hydration of the Chloride Ion in Concentrated Aqueous Solutions Using Neutron Scattering and Molecular Dynamics. Mol. Phys. 2014, 8976, 1-11.

(42) Caminiti, R.; Licheri, G.; Piccaluga, G.; Pinna, G. X-Ray Diffraction Study of $\mathrm{MgCl}_{2}$ Aqueous Solutions. J. Appl. Cryst. 1979, 12, 34-38.

(43) Palinkas, G.; Radnai, T.; Dietz, W.; Szasz, G. I.; Heinzinger, K. Hydration Shell Structures in an $\mathrm{MgCl}_{2}$ Solution from X-Ray and MD Studies. Z. Naturforsch. 1982, 37, 1049-1060.

(44) Bruni, F.; Imberti, S.; Mancinelli, R.; Ricci, M. A. Aqueous Solutions of Divalent Chlorides: Ions Hydration Shell and Water Structure. J. Chem. Phys. 2012, 136, 064520.

(45) Vandevondele, J.; Krack, M.; Mohamed, F.; Parrinello, M.; Chassaing, T.; Hutter, J. QUICKSTEP: Fast and Accurate Density Functional Calculations Using a Mixed Gaussian and Plane Waves Approach. Comput. Phys. Comm. 2005, 167, 103-128.

(46) Bussi, G.; Donadio, D.; Parrinello, M. Canonical Sampling Through Velocity Rescaling. J. Chem. Phys. 2007, 126, 014101.

(47) Becke, A. D. Density-Functional Exchange-Energy Approximation with Correct Asymptotic Behavior. Phys. Rev. A 1988, 38, 3098-3100. 
(48) Lee, C.; Yang, W.; Parr, R. G. Development of the Colle-Salvetti Correlation-Energy Formula into a Functional of the Electron Density. Phys. Rev. B 1988, 37, 785-789.

(49) Grimme, S.; Antony, J.; Ehrlich, S.; Krieg, H. A Consistent and Accurate Ab Initio Parametrization of Density Functional Dispersion Correction (DFT-D) for the 94 Elements H-Pu. J. Chem. Phys. 2010, 132, 154104.

(50) Smith, D. G. A.; Burns, L. A.; Patkowski, K.; Sherrill, C. D. Revised Damping Parameters for the D3 Dispersion Correction to Density Functional Theory. J. Phys. Chem. Lett. 2016, 7, 2197-2203.

(51) Becke, A. D.; Johnson, E. R. A Density-Functional Model of the Dispersion Interaction. J. Chem. Phys. 2005, 123, 154101.

(52) Grimme, S. Semiempirical GGA-Type Density Functional Constructed with a Long-Range Dispersion Correction. J. Comput. Chem. 2006, 27, 1787-1799.

(53) VandeVondele, J.; Hutter, J. Gaussian Basis Sets for Accurate Calculations on Molecular Systems in Gas and Condensed Phases. J. Chem. Phys. 2007, 127, 114105.

(54) Goedecker, S.; Teter, M.; Hutter, J. Separable Dual-Space Gaussian Pseudopotentials. Phys. Rev. B 1996, 54, 1703-1710.

(55) Bleuzen, A.; Helm, L.; Merbach, E. Water Exchange on Magnesium(II) in Aqueous Solution: A Variable Temperature and Pressure. Magn. Reson. Chem. 1997, 35, 765-773.

(56) Kumar, S.; Rosenberg, J. M.; Bouzida, D.; Swendsen, R. H.; Kollman, P. A. Multidimensional Free-Energy Calculations Using the Weighted Histogram Analysis Method. J. Comput. Chem. 1995, 16, 1339-1350.

(57) Paschina, G.; Piccaluga, G.; Pinna, G.; Magini, M. Chloro-Complexes Formation in a $\mathrm{ZnCl}_{2^{-}}$ $\mathrm{CdCl}_{2}$ Aqueous Solution: An X-Ray Diffraction Study. J. Chem. Phys. 1983, 78, 5745.

(58) Irish, D. E.; McCarroll, B.; Young, T. F. Raman Study of Zinc Chloride Solutions. J. Chem. Phys.. 1963, 39, 3436-3444. 
(59) Caminiti, R.; Licheri, G.; Piccalljga, G.; Pinna, G. X-Ray Diffraction Study of a Three-Ion Aqueous Solution. Chem. Phys. Lett. 1977, 47, 275-278.

(60) A. K. Dorosh and A. F. Skryshevskii, The Structural Characteristics of the Immediate Environment of Cations in Aqueous Solutions. Zh. Strukt. Khim. 1964, 5, 911-913.

(61) D’Angelo, P.; Barone, V.; Chillemi, G.; Sanna, N.; Meyer-Klaucke, W.; Pavel, N. V. Hydrogen and Higher Shell Contributions in $\mathrm{Zn}^{2+}, \mathrm{Ni}^{2+}$, and $\mathrm{Co}^{2+}$ Aqueous Solutions: An X-Ray Absorption Fine Structure and Molecular Dynamics Study. J. Am. Chem. Soc. 2002, 124, $1958-1967$.

(62) Kuzmin, A.; Obst, S.; Purans, J. X-Ray Absorption Spectroscopy and Molecular Dynamics Studies of Zn2+ Hydration in Aqueous Solutions. J. Phys. Condens. Matter 1997, 9, 1006510078.

(63) Lightstone, F. C.; Schwegler, E.; Hood, R. Q.; Gygi, F.; Galli, G. A First Principles Molecular Dynamics Simulation of the Hydrated Magnesium Ion. Chem. Phys. Lett. 2001, 343, 549-555.

(64) Riahi, S.; Roux, B.; Rowley, C. N. QM/MM Molecular Dynamics Simulations of the Hydration of $\mathrm{Mg}(\mathrm{II})$ and $\mathrm{Zn}(\mathrm{II})$ Ions. Can. J. Chem. 2013, 99, 1-9.

(65) Tommaso, D. D.; de Leeuw, N. H. Structure and Dynamics of the Hydrated Magnesium Ion and of the Solvated Magnesium Carbonates: Insights from First Principles Simulations. Phys. Chem. Chem. Phys. 2010, 12, 894-901.

(66) Ikeda, T.; Boero, M.; Terakura, K. Hydration Properties of Magnesium and Calcium Ions from Constrained First Principles Molecular Dynamics. J. Chem. Phys. 2007, 127, 074503.

(67) Wu, J. C.; Piquemal, J.-P.; Chaudret, R.; Reinhardt, P.; Ren, P. Polarizable Molecular Dynamics Simulation of $\mathrm{Zn}(\mathrm{II})$ in Water Using the AMOEBa Force Field. J. Chem. Theory Comput. 2010, 6, 2059-2070.

(68) Piquemal, J. P.; Perera, L.; Cisneros, G. A.; Ren, P.; Pedersen, L. G.; Darden, T. A. Towards Accurate Solvation Dynamics of Divalent Cations in Water Using the Polarizable AMOEBA Force Field: From Energetics to Structure. J. Chem. Phys. 2006, 125, 054511. 
(69) Ponder, J. W.; Wu, C.; Pande, V. S.; Chodera, J. D.; Schnieders, M. J.; Haque, I.; Mobley, D. L.; Lambrecht, D. S.; Distasio, R. A.; Head-gordon, M. et al. Current Status of the AMOEBA Polarizable Force Field. J. Phys. Chem. B 2010, 114, 2549-2564.

(70) Marzari, N.; Vanderbilt, D. Maximally Localized Generalized Wannier Functions for Composite Energy Bands. Phys. Rev. B 1997, 56, 22. 


\section{TOC Graphic}

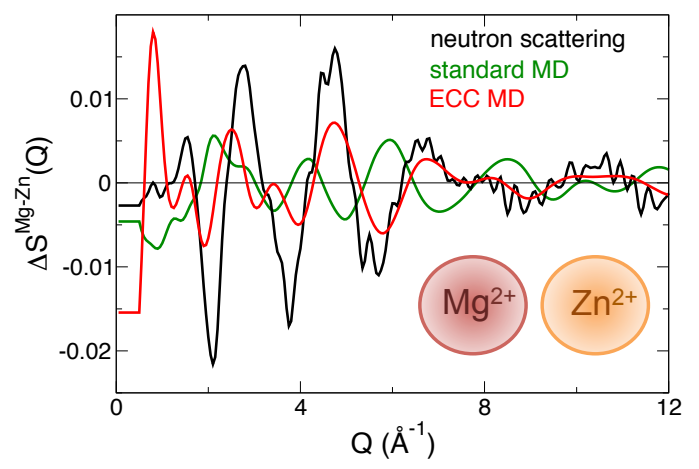

